\title{
An Interval Parameter Calculation Method of Asphalt Pavement Structure Design Based on Point Numerical Algorithm
}

\author{
Limin Tang $\mathbb{D}^{1,2,3}$ and Xiuyan Zhao ${ }^{1,2,3}$ \\ ${ }^{1}$ School of Traffic and Transportation Engineering, Changsha University of Science and Technology, 960 Wanjiali S. Rd., \\ Changsha, Hunan 410004, China \\ ${ }^{2}$ State Engineering Laboratory of Highway Maintenance Technology, Changsha University of Science \& Technology, Changsha, \\ Hunan 410004, China \\ ${ }^{3}$ Co-Innovation Center for Advanced Construction and Maintenance Technology of Modem Transportation Infrastructural \\ Facility, Changsha, Hunan 410004, China
}

Correspondence should be addressed to Limin Tang; tlm@csust.edu.cn

Received 24 August 2020; Revised 15 December 2020; Accepted 16 December 2020; Published 6 January 2021

Academic Editor: Valeria Vignali

Copyright (C) 2021 Limin Tang and Xiuyan Zhao. This is an open access article distributed under the Creative Commons Attribution License, which permits unrestricted use, distribution, and reproduction in any medium, provided the original work is properly cited.

\begin{abstract}
In the asphalt pavement structure design method, the structural analysis and design are generally performed in the form of point values. However, determining the point value form of design parameters based on the statistical analysis theory cannot fully reflect the complex properties such as variability and uncertainty of parameters. In order to further improve the reliability and practicability of pavement design parameters, in this article, we have introduced the interval number representation that can better reflect the complex nature of parameters; but the interval number algorithm is too complicated and common calculation tools and software are difficult to adopt, which limit the wide application of interval analysis to some extent. The article analyzes the algorithm of interval numbers, focusing on the analysis of interval numbers of unary and binary functions. In this way, the point number operation can be used to obtain the interval number result of the function consistent with the interval number algorithm, which avoids the complicated interval number operation process and the interval expansion. The point numerical function algorithm of interval numbers is verified by design parameters and the calculation of asphalt pavement structure such as axle load conversion, cumulative equivalent axis calculation, calculation of foundation layer tensile stress of each structure layer, calculation of mixture penetration strength, fatigue cracking check of asphalt mixture layer, permanent deformation check, and vertical pressure strain test of roadbed top surface. In conclusion, this research provides a simple and easy way to implement the application of mathematical tools for interval analysis, which is suitable for direct use for existing point numerical calculation tools and software.
\end{abstract}

\section{Introduction}

In the current theory and method of pavement structure design, the pavement structure analysis and design are carried out with representative values, i.e., point value mode [1]. Pavement structure design parameters are mostly expressed in the form of point values, such as traffic load parameters (axial load spectrum), fatigue equation parameters, permanent deformation of the asphalt layer, and so on. Pavement structure design parameters are generally obtained by means of testing. Due to the representative problem of sampling of materials, the accuracy and stability of the test equipment, and the objective existence of test data measurement error, the point value of parameters is determined according to the statistical analysis theory and method. It cannot fully reflect the complex nature of parameters such as volatility and uncertainty.

From the quantum effect and the uncertainty principle, the "point true value" of the material parameters of the pavement structure cannot be finalized according to its nature; however, pavement design specifications, including asphalt and cement pavement, and design parameters are 
expressed in terms of point values. From the three stages of pavement engineering design, construction, and acceptance, some indicators are given a range of intervals in acceptance, but in the design and construction process, point values are given. The link between the two is the use of the statistical analysis theory. In fact, the design and construction processes are generally erratic. Therefore, the introduction to the interval number representation instead of the point value is an objective requirement for characterizing the complex nature of the material parameters of the pavement structure and is also consistent with the actual situation where the pavement design deviates and the construction has errors.

Since the emergence of the interval analysis theory, it has received extensive attention and a large number of books have been published on it [2-4]. The articles on interval analysis are increasing, and interval analysis begins to move from theory to practice and plays its role in more and more fields. The important applications of interval analysis in the world include material mechanics, structural mechanics, and so on, which began in the 1980s [5-7].

In the field of engineering, Su et al. [8] studied the reliability analysis method of response surface based on interval variables; Tang et al. [9] proposed the fatigue crack checking process of cement-stabilized macadam is analyzed by using measurement uncertainty and interval analysis theory in order to improve the efficiency of fatigue test data; $\mathrm{Su}$ et al. [10] incorporated a new uncertainty analysis method for engineering structures, the interval analysis method, into the sensitivity analysis of engineering parameters, obtained a new sensitivity analysis method for engineering parameters, and further broadened the theory of interval analysis methods. Application areas: Huang [11] considered the basic parameters that affect the particle size improvement of the roadbed thickness as interval variables. Using interval analysis to study the thickness can better overcome the complexity of the environment and related parameters and the problem of limited information; in the study by Wang et al. [12], starting from data processing, the concept of interval number is given and a multiattribute decision-making model for interval numbers is proposed. The model provides a standardized processing method for the interval number decision matrix. Wang and Tang [13] discussed the influence of different subgrade soil parameters on the subgrade elastic modulus and analyzed the subgrade elastic modulus under equilibrium humidity; Zhang et al. [14] proposed the interval fuzzy evaluation analysis method of highway subgrade stability in a karst area; Liu and Han [15] used fuzzy reliability in the reliability design of the pavement structure, which can further consolidate the performance of the pavement and extend the service life of the pavement; Yu et al. [16] applied the interval analysis model to the stability analysis of slopes. By citing the idea of interval mathematics, the interval limit equilibrium method was used to derive the minimum safety factor interval of the slope, and on this basis, the slope was nonprobabilistically reliable. Degree analysis: Tang and Zheng pointed out that there are two kinds of ill-posed problems in the parameter inversion of the Duncan-Zhang model of the soil and proposed the concepts of interval well-defined and interval ill-posed in parameter inversion; and given the definitions and analysis of interval, the basic theoretical issues of geotechnical engineering were studied and the basic theoretical framework of interval analysis soil mechanics was focused on [17, 18]; Xie et al. [19] introduced the interval analysis method to analyze the fatigue test and data of semirigid base materials and established the fatigue strength equation of semirigid materials in the form of interval parameters; Impollonia and Muscolino [20] proposed a method of evaluating the static response of structures of spaced axial stiffness. An interval analysis method of fatigue crack propagation (FCG) life prediction was proposed by Long et al. [21]. Liu et al. [22] proposed a new static response interval uncertainty analysis method. Sofa et al. [23] proposed an interval finite element analysis method developed by additional unit intervals in the improved interval analysis framework. Dimarogonas [24] proposed an interval analysis of the vibration system. Donald and Chen explored the method of slope stability analysis [25]. Tonon et al. [26] proposed a stochastic set theory about the range of parameters in rock engineering. Giasi et al. [27] studied the fuzzy reliability of the Aliano slope. Schweiger and Peschl [28] proposed the reliability analysis of the stochastic finite element method in geotechnical engineering. For the most part, human factors and environmental conditions affect the measurement results of test parameters. When these digital fuzzy parameters are analyzed in the form of intervals, the accuracy of the measurement results can be more accurately reflected from the aspects of influence analysis, model optimization calculation, and the improvement of the system's predictive ability, so as to draw a new solution [29-36].

However, due to the complexity of the interval algorithm, special interval analysis calculation tools and software are generally used in the calculation of expressions in the form of interval parameters. Because such computing tools and software are not popular, the types are too small and most of them are written by foreign scholars, which greatly limits the learning and use of domestic scholars. Based on this, the article deeply analyzes the algorithm of interval numbers and focuses on the interval number operation of unary and binary functions. By transforming the function expression and other methods, the interval number operation of the function is changed into the upper and lower endpoint value of the independent interval number. In this way, the point numerical operation can be used to obtain the function interval number result consistent with the interval number algorithm, which avoids the complicated interval number operation process and the interval expansion. It can also provide direct application of interval calculation and analysis for various calculation tools and software that currently use point numerical algorithms.

\section{Interval Number Algorithm}

Interval mathematics is a mathematical theory defined on the set of intervals. A continuous subset $X=[\underline{X}, \bar{X}]$ on the set of real numbers $\mathrm{R}$ is called a real interval. $\underline{X}$ is the lower endpoint value of the interval number $X$, and $\bar{X}$ is the upper 
endpoint value of the interval number $X$. An important rule of interval number is $\underline{X} \leq \bar{X}$.

Given $X=[\underline{X}, \bar{X}], Y=[\underline{Y}, \bar{Y}] \in I R$. The following are the four arithmetic rules of interval number:

Interval addition

$$
X+\mathrm{Y}=[\underline{X}+\underline{\mathrm{Y}}, \bar{X}+\overline{\mathrm{Y}}]
$$

Interval subtraction

$$
X-Y=[\underline{X}-\bar{Y}, \bar{X}-\underline{Y}] \text {. }
$$

Interval multiplication

$$
X \times \mathrm{Y}=[\min (\underline{X} \underline{Y}, \underline{X} \bar{Y}, \bar{X} \underline{Y}, \overline{X Y}), \max (\underline{X} \underline{Y}, \underline{X} \bar{Y}, \bar{X} \underline{Y}, \overline{X Y})] .
$$

Interval division

$$
X \div \mathrm{Y}=X \times\left[\frac{1}{\overline{\mathrm{Y}}}, \frac{1}{\underline{\mathrm{Y}}}\right], \quad 0 \notin \mathrm{Y}
$$

Equation (4) shows that for basic interval operations, interval division with 0 is not allowed.

Interval arithmetic has its own characteristics. For example, to find the trigonometric function $\operatorname{Sin}[0, \pi]$. The upper and lower endpoint values of the interval are computed by point numbers, respectively. $\operatorname{Sin}(0)=0, \sin (\pi)=0$, and the upper and lower endpoint values of the obtained function value are all 0 . Is $\operatorname{Sin}[0, \pi]=[0,0]$ ? Surely not! According to the interval number algorithm, $\operatorname{Sin}[0, \pi]=[0,1]$, as shown in Figure 1. Another example is computing Sin [2.6, 7.2]. Using the upper and lower endpoint values calculated by the point numerical algorithm, $\operatorname{Sin}(2.6)=0.5155, \operatorname{Sin}(7.2)=0.7937$, and the interval between the upper and lower endpoint values is $[0.5155,0.7937]$. However, according to the interval number algorithm, $\operatorname{Sin}[2.6,7.2]=[-1,0.9739]$ instead of [0.5155, 0.7937].

In fact, the value of a function of a certain interval variable is the function value field corresponding to the interval variable and is not the interval value obtained by the combination of the endpoint values. For example, the interval variable of $\operatorname{Sin}[0, \pi]$ is $[0, \pi]$, and the corresponding range is $[0,1]$ instead of $[0,0]$, as shown in Figure 1.

Therefore, it is necessary to deeply analyze the algorithm of interval numbers in interval analysis, and especially the interval number algorithm for general functions, because it has a strong practical significance of the correct use of interval analysis.

\section{Point Numerical Operation Method for the Interval Number of Unary Function}

3.1. Calculation Method. Let $f(x)=a x+b$ be a linear onetime linear function, where the independent variable $x$ is the

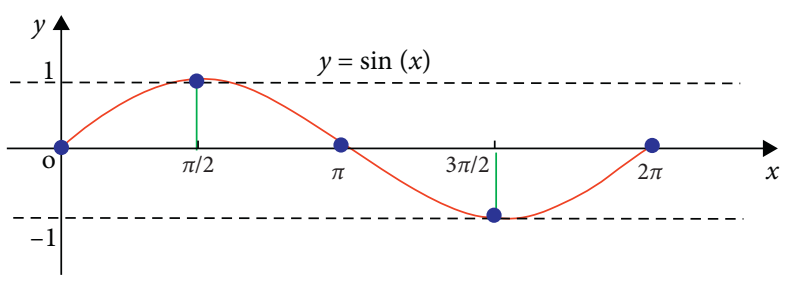

FIgURE 1: Graph of $\sin (x)$ function.

interval number, that is, $x=[\underline{x}, \bar{x}]$, where $\underline{x}$ is the lower endpoint value of the interval number $x$ and $\bar{x}$ is the upper endpoint value of the interval number $x, \underline{x} \leq \bar{x} . a, b \neq 0$.

For the unary linear function $f(x)=a x+b$, if both $a$ and $b$ take the point value and the independent variable $x$ is the interval number, then although $x$ is a positive interval (i.e. $0 \leq \underline{x} \leq \bar{x}$ ), a negative interval (i.e. $\underline{x} \leq \bar{x} \leq 0$ ), or an interval containing 0 (i.e. $\underline{x} \leq 0 \leq \bar{x}$ ), the interval number result of $f(x)$ can be directly calculated by using the upper and lower endpoint values of $x$. It is proved that the derivative $f^{\prime}(x)=a$ of the linear function $f(x)=a x+b$ of one variable is monotonic in any given interval. Thus, $[f(\underline{x}), f(\bar{x})]$ is the value range of the interval $x=[\underline{x}, \bar{x}]$, that is, the interval number result of the unary linear function $f(x)=a x+b$ corresponding to the interval number $x=[\underline{x}, \bar{x}]$ is $[f(\underline{x}), f(\bar{x})]$.

Here, whether or not the interval number $[f(\underline{x}), f(\bar{x})]$ obtained by the point value calculation based on the upper and lower endpoint values of the argument $x=[\underline{x}, \bar{x}]$ is the value range of the $x=[\underline{x}, \bar{x}]$ correspondence function $f(x)$ is a key condition. Due to the monotonicity of the unary function, the number of intervals obtained by the point value based on the upper and lower endpoint values of $x=[\underline{x}, \bar{x}]$ are the value range of $x=[\underline{x}, \bar{x}]$ on the function $f(x)$.

\subsection{Calculation Example}

Example 1. Let $f(x)=3 x+6$, try to calculate the interval number result of the function $f(x)$ of the interval numbers $x_{1}=[-9,-3], x_{2}=[-5,4]$, and $x_{3}=[7,10]$. Using the upper and lower endpoint values, $f(-9)=-21, f(-3)=-3$, $f(-5)=-9, f(4)=18, f(7)=27$, and $f(10)=36$. Then, the result of the $f\left(x_{1}\right)$ interval corresponding to $x_{1}=[-9,-3]$ is $[-21,-3]$; the result of the $f\left(x_{2}\right)$ interval corresponding to $x_{2}=[-5,4]$ is $[-9,18]$; and the result of the $f\left(x_{3}\right)$ interval corresponding to $x_{3}=[7,10]$ is $[27,36]$.

It is noted that

(1) When $a>0$, the number of intervals obtained by the upper and lower endpoint values of the independent variable $x=[\underline{x}, \bar{x}]$ is the function interval number result $[f(\underline{x}), f(\bar{x})]$, that is, the lower endpoint value of $x$ is calculated by the point value to obtain the interval number result. The upper endpoint value of $x$ is calculated by the point value to obtain the upper endpoint value of the interval number result.

(2) When $a<0$, according to the important rule of interval number expression, the lower endpoint value must be less than or equal to the upper endpoint 
value, and then the upper and lower endpoints of the independent variable $x=[\underline{x}, \bar{x}]$ are calculated by the point number, and the function interval number result becomes $[f(\underline{x}), f(\bar{x})]$; that is, the function value calculated from the endpoint value of the independent variable is the lower endpoint value $f(\underline{x})$ of the result of the $f(x)$ interval number, and the function value calculated by the endpoint value $\bar{x}$ of the independent variable is the upper endpoint value $f(\bar{x})$ of the result of the $f(x)$ interval number.

Based on the interval number algorithm, the interval number result of $f(x)$ is obtained, which is consistent with the number of intervals obtained by combining the upper and lower endpoint values.

\subsection{General Conclusion}

(1) A general conclusion is now given. Referring to the nature of higher mathematics for function derivatives, the monotonicity of functions is defined as follows:

Definition 1 (see Ref. [37]). Let function $f(x)$ be a onevariable n-order equation as $f(x)=a x^{n}+b, n>0, x \in D$. If for any two points $\underline{x}, \bar{x} \in D$, when $\underline{x}<\bar{x}$, there is a constant:

(1) $f(\underline{x})<f(\bar{x})$, the function $f(x)$ is said to increase monotonically (strictly) in $D$.

(2) $f(\underline{x})>f(\bar{x})$, the function $f(x)$ is said to be monotonically reduced in $D$ (strictly).

Definition 2 (see Ref. [37]): Set function $f(x)=a x^{n}+b$, $n>0$, continuous on $[\underline{x}, \bar{x}]$, can be guided in $[\underline{x}, \bar{x}]$,

(1) if guided in $[\underline{x}, \bar{x}]$, and $f^{\prime}(x)>0$, then function $f(x)$ monotonically increases on $[\underline{x}, \bar{x}]$

(2) if guided in $[\underline{x}, \bar{x}]$, and $f^{\prime}(x)<0$, then function $f(x)$ monotonically decreases on $[\underline{x}, \bar{x}]$

The interval number operation of the function $f(x)=a x^{n}+b$ satisfying Definitions 1 and 2 can be directly calculated by using the upper and lower endpoints of $x$ to calculate the number of intervals of $f(x)$. At this time, the number of combined intervals of the upper and lower endpoint values is the result of the interval number obtained by the function $f(x)$ through the interval algorithm. It is necessary to consider $a>0$ and $a<0$, the order of combination of the upper and lower endpoint values of the number of intervals calculated by the upper and lower endpoint values of $x$ is different.

(2) Set $f(x)=a x^{n}+b$ to avoid the interval width. Related literature [17] has proved that when the independent variable $x$ appears only once in the expression $f(x)$ on the right side, the interval expansion of the function $f(x)$ result is the interval hyperwidth $=0$, that is, the interval number of $f(x)$ is the value range of the independent variable $f$ in the interval $[x, \bar{x}]$. For example, for $f(x)=x^{2}-2 x+6$, $f(x)=x^{2}+x$, etc., the argument $x$ in the right expression only appears twice, and the interval number of the function result obtained by taking the upper and lower endpoint values of the argument interval is not applicable.

Example 2. Let $f(x)=a x^{n}+b$, try to find the interval number of function $f(x)$ when the interval variable $x=[0,2]$. First, the upper and lower endpoint values are calculated: $f(0)=6$ and $f(2)=10$; therefore, when $f(x)$ is $x=[0,2]$, the result of interval number function calculation is $[6,10]$, which is consistent with the result of using the interval number function operation rule directly.

Example 3. $f(x)=a \times\left(x^{0.22} / b\right)=(a / b) x^{0.22}$, where $a=0.09$ and $b=1$. Try the interval number of $f(x)$ when the interval variable $x=[9949,10551]$ calculated with the upper and lower endpoint values: $0.09 \times 9949^{0.22} \div 1=0.6820 ; 0.09 \times 10551^{0.22} \div 1=0.6908$.

The number of intervals in which the upper and lower endpoint values are combined is [0.6820, 0.6908], which is consistent with the result of the interval number function operation. The calculation code of the interval number function based on the interval analysis calculation tool INTLAB is as follows:

$$
\begin{aligned}
& >>\operatorname{infsup}(0.09 * \operatorname{infsup}(9949,10551) 0.22 / 1) \\
& \text { intval }=[0.6820,0.6908]
\end{aligned}
$$

(3) For other functions such as $f(x)=x^{2}-2 x+6$ and $f(x)=x^{2}+x$, we can transform the expression on the right so that $x$ appears only once, or we can directly use the upper and lower endpoint values to obtain the interval value of the function. If $f(x)=$ $x^{2}-2 x+6$ is rewritten as $f(x)=(x-1)^{2}+5$, when $x=[0,2], x$ is incremented in $[0,1]$ and monotonically decreases, $x$ is monotonically increasing in $[1,2], f(0)=6, f(1)=5$, and $f(2)=6$. When $x=[0,1]$, $f(x)=[5,6]$. When $x=[1,2], f(x)=[5,6]$. Hence, if $x=[0,2]$, then $f(x)=[5,6]$, and this result is consistent with the operation result of the interval number function. Similarly, if $f(x)=x^{2}+x$ is rewritten as $f(x)=(x+(1 / 2))^{2}-(1 / 4)$, the upper and lower endpoint values of the independent variable $x=[\underline{x}, \bar{x}]$ can be used to directly obtain the interval number result of $f(x)$ according to the point numerical function; and also for $f(x)=a x^{n}+b$, when $n<0$, the number of intervals is obtained by directly calculating the upper and lower endpoint values of the independent variable $x=[x, \bar{x}]$ and accordingly the point numerical function needs to be changed; that is, the function value calculated from the endpoint value of the independent variable is the $f(x)$ interval. The function value calculated by the upper end value of the independent variable is the lower end value of the result of interval number $f(x)$, and the function value calculated by the lower end value of the independent variable is the upper 
end value of the result of interval number $f(x)$. Because an important rule of the interval number is the interval numerical expression, $f(\underline{x})<f(\bar{x})$, here, when $n<0, f(\underline{x})$ is calculated by $\bar{x}$ from the point value and $f(\bar{x})$ is calculated by $\underline{x}$. Obviously, when $n>0, f(x)$ is calculated from $\underline{x}$ by point value and $f(\bar{x})$ is calculated by $\bar{x}$.

\section{Point Number Operating Method of the Interval Number of Bivariate Functions and Applications}

4.1. Point Number Operating Method of Interval Number of Bivariate Function. If $X$ and $Y$ in equations (1)-(4) are regarded as binary linear functions, and equations (1)-(4) are the binary-time functions addition, subtraction, multiplication, and division, the specific method of calculating the upper and lower endpoint values is adopted. Investigating equations (1)-(4), the addition rule is easy to understand and remember, but the subtraction method is more complicated. We can calculate the interval number of interval subtraction according to the following steps:

(1) The sign of the subtracted number is unchanged

(2) Add a negative sign to the subtraction, according to the number of intervals, and change the upper and lower endpoints of the interval of the subtraction, that is, $Y=[\underline{Y}, \bar{Y}]$ becomes $-Y=[-\bar{Y},-\underline{Y}]$

(3) Calculate the sum of the upper and lower endpoint values of $X$ and $-Y$ to obtain the number of intervals that are consistent with the calculation results of the interval subtraction rule

Focus on interval multiplication and interval division. Equation (3) is the interval multiplication algorithm, and the lower endpoint value of the interval multiplication calculation result is $\min (\underline{X} \underline{Y}, \underline{X} \bar{Y}, \bar{X} \underline{Y}, \overline{X Y})$, and the upper endpoint value is $\max (\underline{X} \underline{Y}, \underline{X} \bar{Y}, \bar{X} \underline{Y}, \overline{X Y})$. The upper and lower endpoint values of the interval multiplication calculation include a total of four values, namely, $\underline{X} \underline{Y}, \underline{X} \bar{Y}, \bar{X} \underline{Y}$, and $\overline{X Y}$.

(1) First case: $X=[\underline{X}, \bar{X}], Y=[\underline{Y}, \bar{Y}] \in I R, 0 \leq \underline{X} \leq \bar{X}$, $0 \leq \underline{Y} \leq \bar{Y}$

Obviously, $\underline{X} \underline{Y} \leq \underline{X} \bar{Y}, \bar{X} \underline{Y} \leq \overline{X Y}$; at this time, no matter if $\underline{X} \bar{Y}$ and $\bar{X} \underline{Y}$ are big or small; in the four values $\underline{X} \underline{Y}, \underline{X} \bar{Y}, \bar{X} \underline{Y}, \overline{X Y}$, the smallest is $\underline{X} \underline{Y}$ and the largest is $\overline{X Y}$. In this case, when all the interval parameters are greater than 0 , it is very convenient to use the interval endpoint value to calculate the interval number.

(2) Second case: $X=[\underline{X}, \bar{X}] Y=[\underline{Y}, \bar{Y}] \in I R, \underline{X} \leq \bar{X} \leq 0$, $\underline{Y} \leq \bar{Y} \leq 0$.

Evidently, when the number of the two multiplied intervals is a negative interval, $\overline{X Y} \leq \bar{X} \underline{Y}, \underline{X} \bar{Y} \leq \underline{X} \underline{Y}$, no matter which is $X \bar{Y}$ and $\bar{X} Y$ and which is small; in the four values $\underline{X} \underline{Y}, \underline{X} \bar{Y}, \bar{X} \underline{Y}, \overline{X Y}$, the smallest is $\overline{X Y}$ and the largest is $\underline{X} \underline{Y}$. We can directly use the lower endpoint value of $X$ to multiply the lower endpoint value of $Y$ in order to obtain the upper endpoint value of the interval number; multiply the upper endpoint value of $X$ by the upper endpoint value of $Y$ to obtain the lower endpoint value of the interval number.

(3) Third case: $\quad X=[\underline{X}, \bar{X}], Y=[\underline{Y}, \bar{Y}] \in I R, \underline{X} \leq$ $\bar{X}, \underline{Y} \leq \bar{Y}$.

The value of the four endpoints $\underline{X}, \bar{X}, \underline{Y}$, and $\bar{Y}$ is either positive or negative. Among the four values $\underline{X} \underline{Y}, \underline{X} \bar{Y}, \bar{X} \underline{Y}, \overline{X Y}$, the judgment of the size relationship between them is complicated by the existence of the sign, such as $[-3,2] \times[-5,-4]=[-10$, $15]$ and $[-3,2] \times[-5,4]=[-12,15]$; that is, $\overline{X Y}$ and $\underline{X} \underline{Y}$ are not necessarily the largest and smallest of the four values, and it is possible that $\underline{X} \bar{Y}$ and $\bar{X} \underline{Y}$ are the largest and lowest of the four values. Because the third case is too complicated to calculate the interval number using the endpoint value, it is recommended to use the INTLAB correlation interval calculation tool to calculate the result directly.

4.2. Engineering Application Examples. In general, in engineering applications, some of the parameters including the equations used to fit the series of parameters are mostly positive intervals. For example, in pavement engineering, the uniaxial compressive strength, four-point bending strength, and splitting strength test results of the cement-stabilized macadam base material can be fitted by an equation containing a natural logarithmic function, such as the following equation:

$$
S_{c}=a+b \ln (t+c) .
$$

In equation (5), $S_{c}$ is strength, $t$ is age, and $a, b$, and $c$ are all fitting parameters. According to the interval four algorithm, the two interval numbers are added together, and the upper and lower endpoint values can be directly added to obtain the interval number result. The interval number calculation formula of equation (6) is

$$
\begin{aligned}
{\left[\underline{S_{c}}, \overline{S_{c}}\right] } & =[\underline{a}, \bar{a}]+[\underline{b} \ln (\underline{t}+\underline{c}), \bar{b} \ln (\bar{t}+\bar{c})] \\
& =[\underline{a}+\underline{b} \ln (\underline{t}+\underline{c}), \bar{a}+\bar{b} \ln (\bar{t}+\bar{c})] .
\end{aligned}
$$

The key is to check whether $[\underline{b} \ln (\underline{t}+\underline{c}), \bar{b} \ln (\bar{t}+\bar{c})]$ can be the number of intervals in which the upper and lower endpoint values are calculated to obtain the result.

If $f(x)=\ln (t+x)$ is set, then its derivative $f^{\prime}(x)=1 /(t+x)$. When $f^{\prime}(x)=0$, that is, $x=-t$, the function $\ln (t+x)$ has opposite properties on both sides of $x=-t$. Therefore, the interval including $-\mathrm{t}$ needs to be decomposed into two intervals, namely, $[\underline{x},-t]$ and $[-t, \bar{x}]$. The number of intervals of $\ln (t+x)$ can be calculated by using the upper and lower endpoint values.

For example, $\ln (7+[4,6])=[\ln (7+4)$, $\ln (7+6)]=[\ln 11, \ln 13]=[2.3979,2.5649]$ is consistent with the results calculated by the interval analysis calculation tool INTLAB.

If $a, b$, and $c$ are positive intervals, $\left[\underline{S}_{c}, \bar{S}_{c}\right]$ can be directly calculated using the upper and lower endpoint values of $a, b$, and $c$. 
For example, $\left[\underline{S}_{c}, \bar{S}_{c}\right]=[1.2,1.4]+[2.3,2.6] \times \ln (90+$ $[5.1,5.3])=[1.2+2.3 \times \ln (90+5.1), 1.4+2.6 \times \ln \quad(90+$ $5.3)]=[11.68,13.25]$, which is consistent with the results calculated using the INTLAB tool.

Consider a more general case of a binary function: assume $f(x, y)=x^{a} y^{b}$, where $x, y>0$ and $a, b>0$. Obviously, $f(x, y)$ in this case $-x^{a}$ and $y^{b}$ are both increasing functions-satisfies the first case of interval multiplication. Therefore, the upper and lower endpoint values of $x, y$ and $a, b$ can be directly used to calculate the interval number of $f(x, y)$, which is

$$
[\underline{f}(x, y), \bar{f}(x, y)]=\left[\underline{x}^{\underline{a}} \underline{y}^{\underline{b}}, \bar{x}^{\bar{a}} \bar{y}^{\bar{b}}\right] .
$$

In equation (7), it is assumed that $x, y>0$ and $a, b>0$. For example, $f(x, y)=[2.1,5.3]^{[0.22,0.24]} \times \quad[5.2$, $6.1]^{[0.08,0.09]}=\left[2.1^{0.22} \times 5.2^{0.08}, 5.3^{0.24} \times 6.1^{0.08}\right]=$ $[1.3433,1.7559]$, which is consistent with the results calculated using the INTLAB tool.

If $f(x, y)=x^{a}+y^{b}$ and $x, y>0, a, b>0$, the number of intervals of $f(x, y)$ can also be calculated directly by using the upper and lower endpoint values of $x, y$ and $a, b$, which is

$$
[\underline{f}(x, y), \bar{f}(x, y)]=\left[\underline{x}^{\underline{a}}+\underline{y}^{\underline{b}}, \bar{x}^{\bar{a}}+\bar{y}^{\bar{b}}\right] .
$$

The division of the binary function can be changed into the multiplication of binary function by changing the formula. Referring to the above, the interval number results can be directly calculated by using the upper and lower endpoint values. For example, in the early fatigue damage research, the Miner linear model is an earlier theory, and its basic structure is as follows (9):

$$
D(N)=\frac{N}{N_{f}},
$$

where $N_{f}$ is the fatigue life and $N$ is the number of loads. Equation (9) is rewritten into equation (10):

$$
D(N)=N \times \frac{1}{N_{f}},
$$

where $\left[N_{f}, \overline{N_{f}}\right]$ of equation (9) is rewritten as $\left[1 / \overline{N_{f}}, 1 / \overline{N_{f}}\right]$ instead of $\left[1 / N_{f}, 1 / \overline{N_{f}}\right]$.

\section{Point Number Operating Method of the Interval Number of Multivariate Functions}

For ternary function expressions, let $f(x, y, z, \cdots)=x^{a} y^{b} z^{c}, \cdots, x, y, z, \cdots>0, a, b, c \cdots>0$. Obviously, in this case, $f(x, y, z, \cdots)$ is an increasing function, which satisfies the first case of interval multiplication, so the upper and lower endpoint values of $x^{a}, y^{b}, z^{c}, \cdots$ can be directly used to calculate the interval number of $f(x, y, z, \cdots)$, which is

$$
[\underline{f}(x, y, z, \cdots), \bar{f}(x, y, z, \cdots)]=\left[x^{\underline{a}} y^{\underline{b}} z^{\underline{c}}, \cdots, \bar{x}^{\bar{a}} \bar{y}^{\bar{b}} \bar{z}^{\bar{c}}, \cdots\right] .
$$

If

$$
f(x, y, z, \cdots)=x^{a}+y^{b}+z^{c}+\cdots
$$

and $x, y, z, \cdots>0, a, b, c, \cdots>0$, you can also directly use the upper and lower endpoint values of $x, y, z, \cdots, a, b, c, \cdots$ to calculate the interval number of $f(x, y, z, \cdots)$, which is

$$
[\underline{f}(x, y, z, \cdots), \bar{f}(x, y, z, \cdots)]=\left[\underline{x}^{\underline{a}}+\underline{y}^{\underline{b}}+\underline{z}^{\underline{c}}+\cdots, \bar{x}^{\bar{a}}+\bar{y}^{\bar{b}}+\bar{z}^{\bar{c}}+\cdots\right] .
$$

The division of multivariate functions can be transformed into the multiplication of multivariate functions by changing their formulas. Referring to the above, the results of the interval numbers can be directly calculated by using the upper and lower endpoints.

\section{Examples of Asphalt Pavement Structure Design and Checking Calculation}

The basic data of asphalt pavement structure design, namely traffic volume data, are very different from the theoretical prediction. It is unreasonable to use the point numerical traffic volume to design the asphalt pavement structure. It is possible to consider the number of traffic intervals to design the pavement structure.

6.1. Axle Load Conversion and Accumulated Equivalent Axis Interval Number Calculation. The axle load conversion at each level uses the following equation:

$$
N_{1}=\sum_{i=1}^{k} c_{1} c_{2} n_{i}\left(\frac{p_{i}}{p}\right)^{4.35} .
$$

$N_{1}$ is the standard axle load equivalent axle number, times/day; $n_{i}$ is the converted vehicle axle load action number at all levels, times/day; $p$ is the standard axle load, $\mathrm{kN} ; p_{i}$ is the converted vehicle axle load at all levels, $\mathrm{kN} ; k$ is the converted vehicle type; $c_{1}$ is the axle number coefficient, $c_{1}=1+1.2(m-1)$, and $m$ is axle number. When the shaft spacing is greater than $3 \mathrm{~m}$, the calculation is based on a single axle load. When the shaft spacing is less than $3 \mathrm{~m}$, the axle coefficient should be considered; $c_{2}$ is the the wheel group coefficient, and the single-wheel group is 6.4, the twowheel group is 1.0, and the four-wheel group is 0.38 .

As we all know, $n_{i}$, the number of axle loads converted into vehicles at all levels, is obtained directly from the traffic flow observation data. Due to the observation of traffic flow, there are various statistical methods, such as manual, electronic, and toll stations. There are more or less statistical errors in these statistical methods. The traditional calculation of $n_{i}$ in the form of point values cannot fully reflect the complex characteristics of traffic flow. If the statistical error of a certain class of axle load traffic (vehicle/day) is $\pm 1 \%$, then the traffic volume interval parameter is $\left[n_{i}-n_{i} \times 1 \%, n_{i}+n_{i} \times 1 \%\right]$.

According to the point numerical operation method of the interval number of the unary function in the second section, equation (13) directly calculates the lower endpoint value of the equivalent axis sub- $N_{1}$ interval number of the standard axle load using the lower endpoint value $n_{i}-n_{i} \times$ 
$1 \%$ and calculates the standard using the upper endpoint value $n_{i}+n_{i} \times 1 \%$. The upper endpoint value of the number of equivalent $N_{1}$ subintervals of the axle load, assuming the axle number coefficient $c_{1}$ is 1 , the wheel group coefficient $c_{2}$ is $1, p_{i}$ is $55.1 \mathrm{kN}, p$ is $100 \mathrm{kN}$ of the standard axle load, vehicle type $k$ is 1 , and $n_{i}$ is 1000 times a day, is calculated as follows:

$$
\begin{aligned}
N_{1} & =\left[1 \times 1 \times(1000-1000 \times 1 \%) \times\left(\frac{55.1}{100}\right)^{4.35}, 1 \times 1 \times(1000+1000 \times 1 \%) \times\left(\frac{55.1}{100}\right)^{4.35}\right] \\
& =[74.07,75.57] \text { times/day. }
\end{aligned}
$$

Thus, the formula for calculating the number of intervals for the cumulative equivalent axis $N_{e}$ is

$$
\left[\underline{N_{e}}, \overline{N_{e}}\right]=\frac{\left[(1+\gamma)^{t}-1\right] \times 365}{\gamma}\left[\underline{N_{1}}, \overline{N_{1}}\right] \eta \text {. }
$$

$N_{e}$ is the cumulative equivalent axle number; $\eta$ is the lane coefficient value according to specifications; $t$ is the design years; $\gamma$ is the design years, the annual average growth rate of traffic volume forecast; and $N_{1}$ is the standard axle load equivalent axle number, next/day. Now, if $\left[N_{1}, \overline{N_{1}}\right]=[4780.9,4877.9], \gamma=0.055, t=15$, and $\eta=0.5$, according to the second section, equation (15) can directly calculate the equivalent axis subrange $N_{e}$ of the standard axle load using the lower endpoint value $N_{1}$. The lower endpoint value of the number is calculated using the upper endpoint value $\overline{N_{1}}$ to obtain the upper endpoint value of the equivalent axis sub- $N_{e}$ interval number of the standard axle load. After calculation, $\left[N_{e}, \overline{N_{e}}\right]=[1.9551,1.9949] \times 10^{7}$.

6.2. Interval Number Calculation of Structural Coefficient of Tensile Strength. The road grade coefficient $A_{c}$ is 1.0 , the surface layer is asphalt concrete, the surface type coefficient $A_{s}$ is 1.0 , the semirigid base course is 1.0, and the pavement structure type coefficient $A_{b}$ is 1.0 . For the asphalt concrete surface, the interval number of the structural coefficient of tensile strength is calculated as follows:

$$
\left[\underline{K_{s}}, \overline{K_{s}}\right]=0.09 \times \frac{\left[\underline{N_{e}^{\prime}}, \overline{N_{e}^{\prime}}\right]^{0.22}}{A_{c}}
$$

The stable aggregates for inorganic binders:

$$
\left[\underline{K_{s}}, \overline{K_{s}}\right]=0.35 \times \frac{\left[\frac{N_{e}^{\prime}}{N_{e}^{\prime}}\right]^{0.11}}{A_{c}}
$$

For inorganic binder stabilized fine-grained soils:

$$
\left[\underline{K_{s}}, \overline{K_{s}}\right]=0.45 \times \frac{\left[\frac{N_{e}^{\prime}}{N_{e}^{\prime}}\right]^{0.11}}{A_{c}} .
$$

In equations (16)-(18), $N_{e}, N_{e}^{\prime}$-cumulative equivalent axes. Among them, $N_{e}^{\prime}$ is the cumulative equivalent axis for calculating the bending and tensile stresses at the bottom of the semirigid base course. From Section 2, Example 3, we can see that equations (16)-(18) directly calculate the lower endpoint value $K_{s}$ of the equivalent axle number $K_{s}$ interval of the standard axle load using the lower endpoint value $N_{e}$, $N_{e}^{\prime}$ and calculate the upper endpoint value $\overline{K_{s}}$ of the equivalent axle number $K_{s}$ interval of the standard axle load using the upper endpoint value $\overline{N_{e}}, \overline{N^{\prime}}$.

6.3. Calculation of the Number of Tensile Stress Intervals in Each Layer of the Material. The formula for calculating the allowable tensile stress of the pavement structural layer material is

$$
\sigma_{R}=\frac{\sigma_{s}}{K_{s}}
$$

$\sigma_{R}$ is the allowable tensile stress of pavement structural layer materials, $\mathrm{MPa}$; ultimate tensile strength of $\sigma_{s}$ is the structure layer material, $\mathrm{MPa}$, determined by experiment; and structural coefficient of $K_{s}$ is the tensile strength.

For the asphalt concrete surface layer, if the tensile strength structural coefficient is the interval number, then

$$
\left[\underline{\sigma_{R}}, \overline{\sigma_{R}}\right]=\frac{\sigma_{s}}{\left[\underline{K_{s}}, \overline{K_{s}}\right]} .
$$

For the calculation of equation (20), one thing to note is since $\left[K_{s}, \overline{K_{s}}\right]$ is a divisor, it needs to be rewritten as

$$
\left[\underline{\sigma_{R}}, \overline{\sigma_{R}}\right]=\sigma_{s} \times \frac{1}{\left[\underline{K_{s}}, \overline{K_{s}}\right]}=\sigma_{s} \times\left[\frac{1}{K_{s}}, \frac{1}{\overline{K_{s}}}\right] .
$$

For the calculation of equation (21), the lower endpoint value $\sigma_{R}$ of the allowable tensile stress $\sigma_{R}$ interval number can be directly calculated by using the lower endpoint value $1 / \overline{K_{s}}$ and the upper endpoint value $\overline{\sigma_{R}}$ of the allowable tensile stress $\sigma_{R}$ interval number can be calculated by the upper endpoint value $1 / K_{s}$.

$\sigma_{s}$ is the ultimate tensile strength of the structural layer material. According to the relevant technical regulations of the test, the tensile strength test results shall be provided in accordance with the National Standard of the People's Republic of China, "Measurement Uncertainty Evaluation and Expression" (GB/T 27418-2017). The degree of certainty report if the result of the tensile strength test including the uncertainty is the interval number $\left[\sigma_{s}, \overline{\sigma_{s}}\right]$, then equation (21) becomes 


$$
\left[\underline{\sigma_{R}}, \overline{\sigma_{R}}\right]=\left[\underline{\sigma_{s}}, \overline{\sigma_{s}}\right] \times\left[\frac{1}{K_{s}}, \frac{1}{\overline{K_{s}}}\right]=\left[\frac{\sigma_{s}}{\overline{\overline{K_{s}}}}, \frac{\overline{\sigma_{s}}}{K_{s}}\right] .
$$

Table 1 shows the calculation results of the allowable tensile stress design interval for each structural layer of a road surface.

6.4. Interval Number Calculation of Asphalt Mixture Penetration Strength. The penetration strength of asphalt mixture of inorganic binder stabilized base asphalt pavement, asphalt binder stabilized subbase asphalt pavement, and cement concrete base asphalt pavement should meet the following requirements:

$$
R_{\tau s}=\left(\frac{0.31 \lg N_{e 5}-0.68}{\lg \left[R_{a}\right]-13.1 \lg T_{d}-\lg \psi_{s}+2.50}\right)^{1.86} .
$$

Specific explanations of the parameters in equation (23) can be found in formula 5.5.8-1 of Highway Asphalt Pavement Design Code (JTG D50-2017). If the cumulative equivalent axis $N_{e 5}$ is considered as interval number $\left[N_{e 5}, \overline{N_{e 5}}\right]$, the formula for calculating the interval number of penetration strength is as follows:

$$
\left[\underline{R_{\tau s}}, \overline{R_{\tau s}}\right]=\left(\frac{0.31 \lg \left[N_{e 5}, \overline{N_{e 5}}\right]-0.68}{\lg \left[R_{a}\right]-13.1 \lg T_{d}-\lg \psi_{s}+2.50}\right)^{1.86} .
$$

According to Section 2, equation (24) can directly calculate the lower endpoint value $R_{\tau s}$ of the penetration strength $R_{\tau s}$ interval number using the lower endpoint value $N_{e 5}$ and the upper endpoint value $\overline{R_{\tau s}}$ of the $R_{\tau s}$ interval

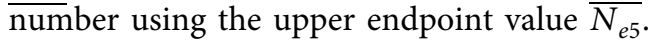

6.5. Fatigue Cracking Check of Asphalt Mixture Layer. The fatigue cracking life of the asphalt mixture should be calculated according to the pavement structure, and the bottom strain of the asphalt mixture layer is calculated according to the following equation:

$$
N_{f 1}=6.32 \times 10^{15.96-0.29 \beta} k_{a} k_{b} k_{T 1}^{-1} \times\left(\frac{1}{\varepsilon_{a}}\right)^{3.97}\left(\frac{1}{E_{a}}\right)^{1.58}(\mathrm{VFA})^{2.72} .
$$

For a detailed explanation of the parameters in equation (25), see the road asphalt pavement design specification (JTG D50-2017) formula B.1.1-1, where $\varepsilon_{a}$ is the bottom strain of the asphalt mixture layer, calculated according to the theory of the elastic layer system. If $\varepsilon_{a}$ is considered to be the interval number $\left[\varepsilon_{a}, \overline{\varepsilon_{a}}\right]$, the interval $\left[N_{f 1}, \overline{N_{f 1}}\right]$ of the fatigue cracking life is calculated as

$$
\left[\underline{N_{f 1}}, \overline{N_{f 1}}\right]=6.32 \times 10^{15.96-0.29 \beta} k_{a} k_{b} k_{T 1}^{-1} \times\left(\frac{1}{\left[\underline{\varepsilon_{a}}, \overline{\varepsilon_{a}}\right]}\right)^{3.97}\left(\frac{1}{E_{a}}\right)^{1.58}(\mathrm{VFA})^{2.72}
$$

At this time, because $\left[\varepsilon_{a}, \overline{\varepsilon_{a}}\right]$ is in the molecular position of fraction, i.e., a divisor, the lower point value $N_{f 1}$ of the fatigue crack life interval number cannot be calculated directly by using the lower point value $\varepsilon_{a}$ and the upper end value $\overline{N_{f 1}}$ of the fatigue crack life interval number can be calculated by using the upper end value $\overline{\varepsilon_{a}}$.

According to the interval division calculation rule, combined with the third section of the article and equation (10), equation (26) can be rewritten as

$$
\left[\underline{N_{f 1}}, \overline{N_{f 1}}\right]=6.32 \times 10^{15.96-0.29 \beta} k_{a} k_{b} k_{T 1}^{-1} \times\left(\left[\overline{\overline{\varepsilon_{a}}}, \frac{1}{\varepsilon_{a}}\right]\right)^{3.97}\left(\frac{1}{E_{a}}\right)^{1.58}(\mathrm{VFA})^{2.72}
$$

At this time, the lower endpoint value $N_{f 1}$ of the fatigue cracking life interval number can be directly calculated by using the lower endpoint value $1 / \overline{\varepsilon_{a}}$ and the upper endpoint value $\overline{N_{f 1}}$ of the fatigue cracking life interval number can be calculated by using the upper endpoint value $1 / \varepsilon_{a}$.

6.6. Verification of Permanent Deformation of Asphalt Mixture Layer. According to the rutting test under standard conditions, the permanent deformation of each layer of asphalt mixture in rutting test is obtained, and the permanent deformation of each layer and the total permanent deformation of asphalt mixture layer are calculated according to the formula B.3.2-1 of highway asphalt pavement design code (JTG D50-2017). The formula for calculating the permanent is as follows:

$$
R_{a i}=2.31 \times 10^{-8} k_{R i} T_{p e f}^{2.93} p_{i}^{1.80} N_{e 3}^{0.48}\left(\frac{h_{i}}{h_{0}}\right) R_{0 i} .
$$

Specific explanations of the parameters in the formula can be found in formula B.3.2-1 of Highway Asphalt 
TABLE 1: Number of allowable tensile stress design intervals for each layer of pavement.

\begin{tabular}{lcr}
\hline Material name & $h(\mathrm{~cm})$ & Allowable tensile stress $(\mathrm{MPa})$ \\
\hline Fine-grained asphalt concrete & 4 & {$[0.3826,0.3899]$} \\
Medium granular asphalt concrete & 6 & {$[0.2725,0.2793]$} \\
Coarse asphalt concrete & 8 & {$[0.2174,0.2240]$} \\
Cement gravel & 25 & {$[0.2233,0.2331]$} \\
Lime soil & 40 & {$[0.0779,0.0853]$} \\
Soil base & - & - \\
\hline
\end{tabular}

Pavement Design Code (JTG D50-2017). If the cumulative equivalent axis $N_{e 3}$ is the interval number $\left[N_{e 3}, \overline{N_{e 3}}\right]$, the calculation formula for the $i$-layer permanent deformation interval number is

$$
\left[\underline{R_{a i}}, \overline{R_{a i}}\right]=2.31 \times 10^{-8} k_{R i} T_{p e f}^{2.93} p_{i}^{1.80} \times\left(\underline{N_{e 3}}, \overline{N_{e 3}}\right)^{0.48}\left(\frac{h_{i}}{h_{0}}\right) R_{0 i} .
$$

According to the second section, equation (29) can directly calculate the lower endpoint value $R_{a i}$ of the $i$-layer permanent deformation amount $R_{a i}$ interval using the lower endpoint value $N_{e 3}$ and the upper endpoint value $\overline{R_{a i}}$ of the $R_{a i}$ interval number using the upper endpoint value $\overline{N_{e 3}}$.

6.7. Vertical Compressive Strain Check on the Top Surface of the Subgrade. According to the following formula, vertical compressive strain check on the top surface of the subgrade is

$$
\left[\varepsilon_{z}\right]=1.25 \times 10^{4-0.1 \beta}\left(k_{T 3} N_{e 4}\right)^{-0.21} .
$$

Specific explanations of the parameters in equation (30) can be found in formula B.4.1 of Highway Asphalt Pavement Design Code (JTG D50-2017). If the cumulative equivalent axis $N_{e 4}$ is the interval number $\left[N_{e 4}, \overline{N_{e 4}}\right]$, the formula for calculating the allowable vertical compressive strain interval of the top surface of the subgrade is as follows:

$$
\left[\varepsilon_{z}\right]=1.25 \times 10^{4-0.1 \beta}\left(k_{\text {Т3 }}\right)^{-0.21}\left(\left[N_{e 4}, \overline{N_{e 4}}\right]\right)^{-0.21} .
$$

Since the index of $N_{e 4}$, that is, -0.21 , is negative, it needs to be rewritten as

$$
\left[\underline{\varepsilon_{z}}, \overline{\varepsilon_{z}}\right]=1.25 \times 10^{4-0.1 \beta}\left(k_{T 3}\right)^{-0.21}\left(\left[\frac{1}{\overline{N_{e 4}}}, \frac{1}{N_{e 4}}\right]\right)^{0.21} .
$$

After this, according to the second section, equation (32) can directly calculate the lower endpoint value $\varepsilon_{z}$ of the allowable vertical compressive strain $\varepsilon_{z}$ interval number of the top surface of the subgrade using the lower endpoint value $1 / \overline{N_{e 4}}$ and the upper endpoint value of the number $\overline{\varepsilon_{z}}$ of the $\varepsilon_{z}$ interval using the upper endpoint value $1 / N_{e 4}$.

In addition to the penetration strength of the asphalt mixture, fatigue cracking check, permanent deformation check, vertical pressure, and strain test of the roadbed top surface, the new road asphalt pavement design specification also stipulates subgrade elastic modulus, asphalt surface low temperature cracking index, antifreeze, design parameters, and so on and performs indicator checks such as thickness check and acceptance deflection. The specific analysis can be carried out according to the formula. Referring to the methods described in Sections 2-4, the expression is rewritten or some parameters are simplified, and the design parameters and the calculation formulas for the calculation index suitable for directly calculating the interval number of the upper and lower endpoints of the independent variable are established.

\section{Conclusions}

This paper discusses the transformation of the complex interval number function algorithm into the point numerical algorithm and provides a simple and easy interval analysis process and method for the various calculation tools and software that generally use the point numerical algorithm. Taking the calculation and verification of interval parameters of asphalt pavement structure as an example, the theory and method discussed in the article are verified, and the following conclusions are obtained:

(1) According to the upper and lower endpoint values of the function argument $x=[\underline{x}, \bar{x}]$, the number of intervals obtained by the point value operation, if the independent variable $x=[\underline{x}, \bar{x}]$ corresponds to the value range on the function $f(x)$, then the number of intervals obtained using the upper and lower endpoint values of the argument is equal to the interval number of function obtained by the interval number algorithm.

(2) In the unary function, if the argument in the right expression only appears once, and the monotonicity of the function is consistent within the interval of the independent variable, the upper and lower endpoint values of the argument interval can be used to obtain the interval number of the function.

(3) For a binary function, especially when multiplying two independent variables, the positive and negative values of the independent variable interval must be considered. When the first and second cases in Section 3 of this paper are satisfied, the number of intervals can be obtained by calculating the upper and lower endpoint values of the two independent variable intervals.

(4) For the multivariate function, the situation is more complicated, and there are many factors to consider. We can learn this from the example in Section 4 of the article. 
(5) The division of unary, binary, and multivariate functions can be multiplied by the change in the expression, and then the upper and lower endpoint values are used to calculate the interval number as described in this paper.

(6) When calculating the interval number of the function using the upper and lower endpoint values of the independent variable, if the coefficient or the index in front of the independent variable is greater than 0 , and the function expression only adds and multiplies the two operations, the interval number calculation is relatively simple. However, when the coefficient or exponent of the independent variable is less than 0 , and the function expression has two operations of subtraction and division, the interval number calculation is more complicated and needs careful analysis.

In conclusion, this research provides a convenient and easy-to-implement process and method for the popularization of interval analysis theory. In the current analysis and calculation of road mechanics, most of the parameters involved are positive numbers, that is, greater than zero. In addition, the mechanical parameter measurement uncertainty report provided by the national standard "Measurement Uncertainty Evaluation and Representation" (GB/T 27418-2017) is mostly a positive interval value. In practical applications, the data processing software and various other point numerical calculation tools can be directly used to quickly obtain the interval number of parameters, thereby avoiding complicated interval algorithm programming. However, further analysis of the mechanical mechanics of the pavement involves complex operations such as differentiation, integration, and even matrix, especially numerical simulation. In future, how to simplify the calculation of interval numbers in such complex operations is an important issue to be solved.

\section{Data Availability}

The data used to support the findings of this study are available from the corresponding author upon request.

\section{Conflicts of Interest}

The authors declare that there are no conflicts of interest regarding the publication of this paper.

\section{Acknowledgments}

This work was supported by the National Key Research and Development Plan (2017YFC0805300), National Natural Science Foundation of China (51578081), Excellent Youth Project of the Hunan Provincial Department of Education (15B010), and National Engineering Laboratory Open Fund Project of Highway Maintenance Technology (kfj140102).

\section{References}

[1] Chinese Standards Institution, JTG D50, 40:41. Highway Asphalt Pavement Design Specification, Chinese Standards Institution, Beijing, China, 2017.

[2] R. E. Moore, Interval arithmetic and automatic error analysis in digital computing, Thesis ( $\mathrm{PhD})$, Stanford University, Stanford, CA, USA, 1962.

[3] R. E. Moore, Interval Analysis, Prentice-Hall, Upper Saddle River, NJ, USA, 1966.

[4] R. E. Moore, M. J. Cloud, and R. B. Kearfott, Introduction to Interval Analysis, Springer, Berlin, Germany, 2009.

[5] D. Degrauwe, G. Lombaert, and G. De Roeck, "Improving interval analysis in finite element calculations by means of affine arithmetic," Computers \& Structures, vol. 88, no. 3-4, pp. 247-254, 2010.

[6] J. J. Chen, "Interval analysis method for controllability of uncertain systems," Journal of Liaoning University (Natural Science Edition), vol. 2, pp. 57-66, 1981, in Chinese.

[7] L. Q. Qi, "Interval analysis," Journal of Operations Research, vol. 1, pp. 29-35, 1982.

[8] Y. H. Su, M. C. He, and M. H. Zhao, "etc, Reliability analysis of response surface method based on interval variables," Chinese Journal of Geotechnical Engineering, vol. 27, no. 12, pp. 1408-1413, 2005.

[9] L. M. Tang, Y. Xiao, and J. W. Xie, "Fatigue cracking checking of cement stabilized macadam based on measurement uncertainty and interval analysis," Construction and Building Materials, vol. 250, Article ID 118921, 2020.

[10] J. B. Su, G. J. Shao, and W. J. Chu, "Pile sensitivity analysis method of soil parameters based on interval," Applied Mathematics and Mechanics, vol. 29, no. 12, pp. 1502-1512, 2008, in Chinese.

[11] M. K. Huang, "Study on thickness of modified subgrade based on interval analysis," Journal of Chongqing Jiaotong University (Natural Science Edition), vol. 1, pp. 77-79, 2009, in Chinese.

[12] Z. H. Wang, X. C. Wang, and S. B. Ma, "Comprehensive evaluation of pavement performance based on interval number approximation method," Journal of Highway and Transportation, vol. 1, pp. 21-25, 2009, in Chinese.

[13] L. Wang and L. M. Tang, "Calculation method of subgrade resilient modulus based on Interval Parameters," Chinese and Foreign Highways, vol. 38, no. 1, pp. 15-20, 2018.

[14] Y. J. Zhang, W. G. Cao, M.-H. Zhao, and H. Zhao, "Interval fuzzy judgment method for roadbed stability in karst area," Chinese Journal of Geotechnical Engineering, vol. 1, pp. 38-44, 2011.

[15] J. Q. Liu and J. Han, "Reliability analysis of asphalt pavement structure based on fuzzy mathematical theory," Applied Mathematics and Mechanics, vol. 39, no. 9, pp. 1081-1090, 2018.

[16] S. F. Yu, Z. Z. Chen, and M. R. Zhang, "Slope stability analysis based on interval uncertainty analysis method," Journal of Engineering Geology, vol. 2, pp. 228-233, 2012.

[17] L. M. Tang and J. L. Zheng, "Parameter inversion method based on interval posedness and interval ill-posedness theory," China Civil Engineering Journal, vol. 49, no. 11, pp. 91-96, 2016.

[18] L. M. Tang and J. L. Zheng, Interval Analysis of Geotechnical Engineering Theory and Method, Science Press, Beijing, China, 2017.

[19] J. W. Xie, L. M. Tang, and S. T. Lv, "Standardization of fatigue characteristics of cement-treated aggregate base materials under different stress states," Applied Sciences-Basel, vol. 8, no. 9, 2018. 
[20] N. Impollonia and G. Muscolino, "Interval analysis of structures with uncertain-but-bounded axial stiffness," Computer Methods in Applied Mechanics and Engineering, vol. 200, no. 21-22, pp. 1945-1962, 2011.

[21] X. Y. Long, C. Jiang, K. Liu, X. Han, W. Gao, and B. C. Li, “An interval analysis method for fatigue crack growth life prediction with uncertainty," Computers and Structures, vol. 210, pp. 1-11, 2018.

[22] Y. Liu, X. Wang, and L. Wang, "Interval uncertainty analysis for static response of structures using radial basis functions," Applied Mathematical Modelling, vol. 69, pp. 425-440, 2019.

[23] A. Sofa and E. Romeo, "A novel interval finite element method based on the improved interval analysis," Computer Methods in Applied Mechanics and Engineering, vol. 311, pp. 671-697, 2016.

[24] A. D. Dimarogonas, "Interval analysis of vibrating systems," Journal of Sound and Vibration, vol. 183, no. 4, pp. 739-749, 1995.

[25] I. B. Donald and Z. Chen, "Slope stability analysis by the upper bound approach: fundamentals and methods," Canadian Geotechnical Journal, vol. 34, no. 6, pp. 853-862, 1997.

[26] F. Tonon, A. Bernardini, and A. Mammino, "Determination of parameters range in rock engineering by means of random set theory," Reliability Engineering \& System Safety, vol. 70, no. 3, pp. 241-261, 2000.

[27] C. I. Giasi, P. Masi, and C. Cherubini, "Probabilistic and fuzzy reliability analysis of a sample slope near Aliano," Engineering Geology, vol. 67, no. 3-4, pp. 391-402, 2003.

[28] H. F. Schweiger and G. M. Peschl, "Reliability analysis in geotechnics with the random set finite element method," Computers and Geotechnics, vol. 32, no. 6, pp. 422-435, 2005.

[29] S. Chen, H. Lian, and X. Yang, "Interval static displacement analysis for structures with interval parameters," International Journal for Numerical Methods in Engineering, vol. 53, no. 2, pp. 393-407, 2002.

[30] D. M. Do, W. Gao, C. Song, and M. Beer, "Interval spectral stochastic finite element analysis of structures with aggregation of random field and bounded parameters," International Journal for Numerical Methods in Engineering, vol. 108, pp. 1198-1229, 2016.

[31] A. Sofi and E. Romeo, "A unified response surface framework for the interval and stochastic finite element analysis of structures with uncertain parameters," Probabilistic Engineering Mechanics, vol. 54, pp. 25-36, 2016.

[32] R. Santoro, G. Muscolino, and I. Elishakoff, "Optimization and anti-optimization solution of combined parameterized and improved interval analyses for structures with uncertainties," Computers \& Structures, vol. 149, pp. 31-42, 2015.

[33] F. Giunta, G. Muscolino, S. Alba, and I. Elishakoff, "Dynamic analysis of Bernoulli-Euler beams with interval uncertainties under moving loads," Procedia Engineering, vol. 199, pp. 2591-2596, 2017.

[34] Y. Huang, "An interval algorithm for uncertain dynamic stability analysis," Applied Mathematics and Computation, vol. 338, pp. 567-587, 2018.

[35] B. Wu, D. Wu, W. Gao, and C. Song, "Time-variant random interval natural frequency analysis of structures," Journal of Sound and Vibration, vol. 414, pp. 284-298, 2018.

[36] C. Yang, S. Tangaramvong, W. Gao, and F. Tin-Loi, "Interval elastoplastic analysis of structures," Computers \& Structures, vol. 151, pp. 1-10, 2015.

[37] Higher Education Press, Advanced Mathematics, Higher Education Press, Beijing, China, 6th edition, 2007. 